(Aus der Patholog.-anat. Abteilung des Kaiser u. Kaiserin Friedrich-KinderKrankenhauses zu Berlin.)

\title{
Beitrag zur Appendicitisfrage beim Säugling und im frühen Kindesalter.
}

\author{
Von \\ Dr. Rheindorf.
}

Mit 5 Textabbildungen.

(Eingegangen am 22. Juli 1922.)

Fast alle Autoren, die zur Frage der Appendicitis im Säuglingsalter Stellung genommen haben, sind sich darüber einig, daß diese Erkrankung sehr selten ist. In meinem ersten Aufsatze ${ }^{1}$ zur Appendicitisfrage aus dem Jahre 1912 bin ich kurz hierauf eingegangen. Ich erwähnte damals die Angaben von Kirmisson und Guimbellot, die aus der ganzen Literatur 9 beweiskräftige Fälle zusammengestellt hatten, und ich referierte über die Erfahrungen bedeutender Pädiater, z. B. Heubners und Baginskys, die im Laufe ihrer langjährigen Erfahrungen keinen Fall von Appendicitis im Säuglingsalter zu brobachten Gelegenheit hatten.

Praktisch zu demselben Resultate kommt auf Grund reicher Er. fahrungen Finkelstein ${ }^{2}$ ). Dieser Autor erwähnt in seinem 1921 erschienenen Lehrbuche nur einen Fall eigener Beobachtung. Aus der dort erwähnten Dissertation von Bamberg ${ }^{3}$ ) ersehe ich, daß meine oben wiedergegebenen Zahlen von Kirmisson und Guimbellot etwas zu niedrige sind, da es Bamberg schon im Jahre 1905 gelang, 13 sichere Fälle aus der Weltliteratur zusammenzustellen. Ich möchte aber noch kurz larauf hinweisen, daß Bamberg auch die Ansichten anderer Autoren erwähnt, so z. B. Balzers und Fisches, die über ein nicht so seltenes Vorkommen dieser Er krankung berichten. (Siehe auch Schloßmann, Arch. f. Kinderheilkunde 1922.)

Zur Frage dieser entgegengesetzten Ansichten möchte ich in nachfolgenden Ausführungen keine Stellung nehmen, sondern nur angeben, daß ich im Laufe meiner fast 12 jährigen Tätigkeit als Prosektor, wo ich ein reichhaltiges Appendicitismaterial untersuchen konnte, nur einen derartigen Fall zu untersuchen Gelegenheit hatte. Allerdings kam dort noch ein zweiter derartiger Fall zur Operation, den ich dem Vater des betreffenden Säuglings, einem Fachpathologen, zur Untersuchung überließ. Es würde dies also im Sinne der zuerst von mir erwähnten Forscher sprechen, welche die Appendicitis in Säuglingsalter 
für eine sehr seltene Erkrankung halten. Anscheinend zu demselben Resultate kommt auf Grund 1000 untersuchter Wurmfortsätze Aschoff ${ }^{4}$ ), der nur einen Fall bei einem 4 Monate alten Säugling erwähnt. Hier hatte sich die Appendicitis in einem intakten Wurmfortsatze entwickelt.

Da ich diese Frage für eine solche von grundsätzlicher Wichtigkeit halte, und ich nirgends genaue Angaben über den histologischen Befund eben beginnender Appendicitiden im Säuglingsalter finden konnte, so möchte ich etwas genauer über einen von mir untersuchten Fall berichten. Der Fall kam schon im Jahre 1913 in meine Beobachtung und betraf einen 3 Monate alten Säugling, der ambulant wegen einer rechtsseitigen, scheinbar eingeklemmten Leistenhernie operiert wurde. Die Erkrankung hatte plötzlich mit Erbrechen, hohem Fieber und Unruhe eingesetzt und war noch nicht 24 Stunden alt. Für die Überlassung des lebenswarm fixierten Wurmfortsatzes und die klinischen Angaben bin ich Herrn Geh. Rotter sehr zu Dank verpflichtet. Bei der Operation fanden sich im Bruchsack der Blinddarm und der Wurmfortsatz, aber wider Erwarten am Bruchinhalte nicht die geringsten Einklemmungserscheinungen. Nur der Wurmfortsatz zeigte eine ganz geringe Rötung; er wurde entfernt und liebenswürdigerweise für mich lebenswarm in Formalin fixiert, da ich um möglichst normale Wurmfortsätze von Säuglingen gebeten hatte. Die Heilung erfolgte per primam. Der Säugling war gleich nach der Operation entlassen worden, und da der Wurmfortsatz erst einige Wochen später von mir in Serienschnitte zerlegt wurde, kann ich keine Angaben über den kleinen Patienten machen, da ich sonst mit der gütigen Erlaubnis des Herm Geh. Rotter noch spezielle Untersuchungen anstellte oder spezielle Fragen an die Patienten oder bei kleinen Kindern an deren Eltern zu richten pflegte.

Ich habe so lange mit der Veröffentlichung dieses Falles gewartet, weil mir keinerlei Kontrollmaterial zur Verfügung stand, ich ein solches jedoch für wünschenswert hielt, um zu einer einwandfreien Beurteilung der an dem fraglichen Wurmfortsatze vorgefundenen Veränderungen zu gelangen. Nachdem ich nun durch meine Tätigkeit am Kaiser FriedrichKinder-Krankenhause in den Besitz zahlreicher Wurmfortsätze von Säuglingen gelangt bin, möchte ich nicht länger zögern, mit dem von mir schon vor 9 Jahren erhobenen Befunde an die Öffentlichkeit zu treten, da er für das Verständnis der Pathogenese der Appendicitis im Säuglingsalter von prinzipieller Bedeutung ist. Ein Teil meines neuen Untersuchungsmateriales ist durch gleich nach dem Tode vorgenommene intraabdominelle Formalininjektionen lebenswarm fixiert worden. Für dieses freundliche Entgegenkommen spreche ich Herrn Dr. Gehrt und Frl. Dr. Usbeck auch an dieser Stelle meinen verbindlichsten Dank aus. Ohne derartige Formalininjektionen ist natürlich die exakte Beurteilung der ersten bei der Appendicitis vorkommenden Veränderungen unmög- 
lich oder doch jedenfalls sehr unsicher, da die kadaverösen Veränderungen sich sehr störend bemerkbar machen. Besonderśs spielt dies bei geringgradigen Epithelveränderungen eine Rolle, die in erster Linie in Betracht kommen. Der ganz in Serien geschnittene Wurmfortsatz zeigte nun überraschenderweise an verschiedenen Stellen die eben beginnende Eiterung an kleinen Epitheldefekten, und zwar betraf die Lokalisation dieser Epitheldefekte, welch letztere sämtliche Übergänge einer fehlenden Reaktion bis zur ausgesprochenen leukocytären Infiltration zeigten, sowohl die Buchten als auch die Faltenhöhen. Das Lumen ließ jegliche Retention von Kotbestandteilen vermissen, ebenso fanden sich nirgends Abknickungen, Stenosen oder Verwachsungen. Das einzige, was sich im Lumen findet, ist neben etwas Schleim eine mikroskopisch wahrnehmbare mäBige Anzahl von Leukocyten, als deren Auswanderungsort die oben erwähnten an den verschiedensten Stellen der Schleimhaut befindlichen Epitheldefekte festzustellen sind, die verschiedene Grade der leukocytären Auswanderung

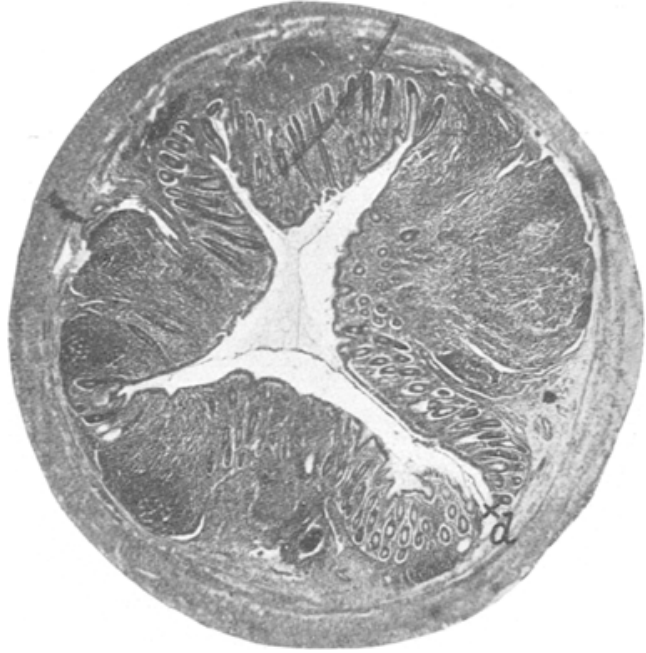

Abb. 1. Processus-Querschnitt. (83 fache VergröBerung. 3 Monate a]ter Säugling.) Die Schleimhaut zeigt intaktes Epithel bis auf eine Stelle bei $x d$ rechts unten in einer Bucht. Hier besteht ein schon bei dieser schwachen VergröBerung herauskommender Epitheldefekt, der bei starker Vergrößerung die beginnende Eiterung zeigt. (Siehe Abb. 4.) Die drei anderen Buchten mit tadellos erhaltenem Epithel. aufweisen, und welche durch alle Übergänge mit reaktionslosen Epitheldefekten zusammenhängend verbunden sind. Neben diesen Leukocyten finden sich noch Fibrinfäden und etwas Schleim im Lumen des Wurmfortsatzes. Entsprechend dem Alter von 3 Monaten findet sich noch keine vollständige Entwicklung des Lymphknötchenapparates, sondern an einigen Stellen sieht man in der Schleimhaut Lymphknötchen, die sich allerdings durch eine erhebliche Größe auszeichnen. Eine Eosinophilie der Schleimhaut fehlt. Entsprechend der wenig ausgebildeten Entwicklung des lymphatischen Apparates ist das Lumen noch relativ weit, wenn es auch je nach der verschiedenen Entwicklung des lymphatischen Apparates auf den einzelnen Querschnittsbildern sehr wechselnde Größenunterschiede auf weist. Die Abb. 1 und 2 mögen zur Illustration dieser Verhältnisse dienen. 
Das Epithel ist überall tadellos erhalten bis auf zwei Stellen, an denen in Abb. 1 in der Tiefe einer Bucht rechts und in Abb. 2 auf einer Faltenhöhe eine eben beginnende leukocytäre Infiltration an den schon bei dieser schwachen Vergrößerung deutlich kenntlichen Epitheldefekten hervortritt. Wenn ich nun noch hinzufüge, daß sich an diesen in den übrigen Schnitten in der Mehrzahl findenden Epitheldefekten, die alle Übergänge einer Reaktionslosigkeit bis zu einer starken leukocytären Infiltration aufweisen, stellenweise auch die verschiedensten Grade der Epithelregenerationen nachweisen lassen, so stelle ich fest, daß wir also

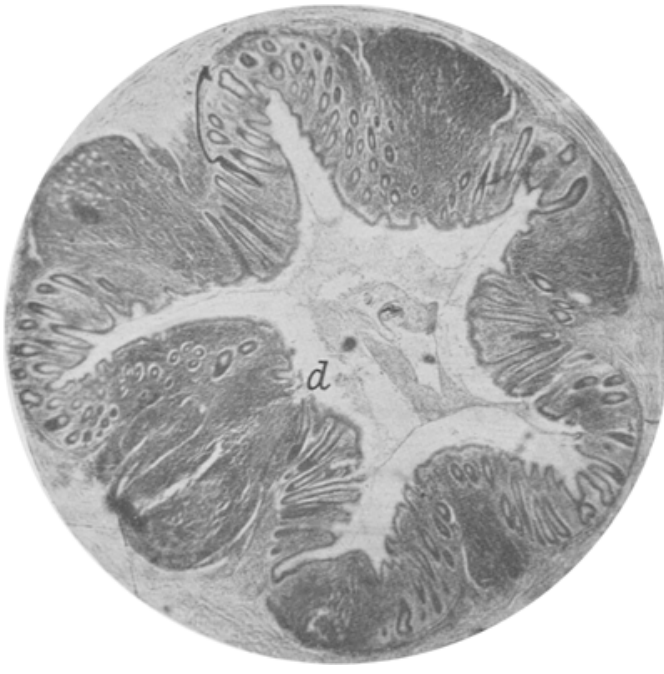

Abb. 2. Processus-Querschnitt. (33 fache Vergrößerung. 3 Monate alter Säugling.) Das Lumen ist sehr weit. Das Epithel ist intakt bis auf die Stelle bei $d$ auf einer Faltenhöhe. Hier findet sich ein kleiner Epitheldefekt mit einem Leukocytenpfropf. Die leukocytäre Infiltration reicht bis in das darunter befindliche Lymphknötchen. Im Lumen des Processus Schleim mit Leukocyten. in diesem Säuglingswurm fortsatze alle die histologischen Feststellungen machen können, wie ich sie in meiner Monographie $^{5}$ ) für die Pathogenese der Erwachsenen- und Kinder-Appendicitis als größtenteils typisch nachweisen konnte.

Die Abb. 3 zeigt auf einem Längsschnitte an der oberen Seite sehrschön das flache, sich regenerierende Epithel, das an beiden Seiten in zylindrisches Epithel übergeht, während an der gegenüberliegenden Seite der hochzylindrische Epithelsaum sichtbar ist. Das sind also dieselben Veränderungen, wie ich sie an der Hand der Abb. 31, 32, 35 und $36 \mathrm{~m}$. M. zur Anschauung brachte. Diese Regenerationen beweisen, wie ich an der Hand mehrerer Abbildungen zeigte, daß in diesen Wurmfortsätzen, in denen sich eine frische eiterige Entzündung an Epitheldefekten entwickelt, diese Epitheldefekte schon längere Zeit bestanden haben müssen, und diese bei ihrem gesetzmäBigen Vorkommen bei der Mehrzahl der ohne Stenosen, Kotsteine oder Verwachsungen entstehenden Appendicitiden die notwendige Vorbedingung der Eiterung darstellen. Die Entstehung dieser Epitheldefekte führte ich auf die teils toxische, teils mechanisch das Epithel zerstörende Wirkungsweise der Oxyuren zurück, die ich an der Hand der Abb. 2, 3, 4, 5, 6, 7, 8, 9, 10, 16, 17 und 18 in meiner Monographie bewies. In diesem Wurmfortsatze eines 3 Monate alten 
Säuglings ließen sich nun Oxyuren selbst nicht nachweisen, wohl aber fand ich Cuticularmembranen, wie ich sie in Abb. 24. meiner Monographie abbildete und deren Vorkommen ja auch schon von Suzuckib) bestätigt worden ist. Von der Wiedergabe sehe ich aus Sparsamkeitsrücksichten ab. Wenn ich also infolge Fehlens der Oxyuren im Wurmfortsatze selbst nicht in der Lage bin, im histologischen Präparate den exakten Nachweis zu führen, daß die hier vorhandenen Epitheldefekte auf die Wirkungsweise der Oxyuren zurückzuführen sind, so nehme

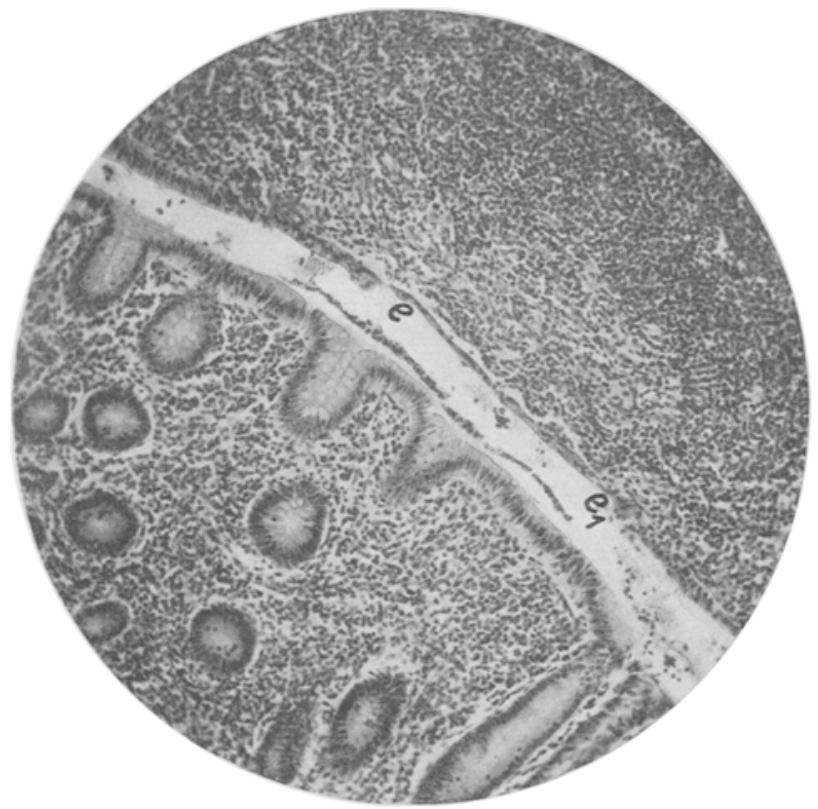

Abb. 3. Processus-Längsschnitt. (300 fache Vergrößerung. 3 Monate alter Säugling.) Fall von frischer, noch nicht 24. Stunden alter Appendicitis. An der unteren Schleimhautseite überall intaktes hochzylindrisches Epithel. An der oberen Schleimhautseite flaches sich regenerierendes Epithel (von $e-e_{1}$ ), das sich beiderseits in zylindrisches Epithel fortsetzt.

ich für sie nach Analogie dieselbe Entstehung an, wie ich sie in meiner Monographie exakt nachwies. Die grundsätzliche Bedeutung dieses Befundes ist einleuchtend. Ebenso wie am Erwachsenen- und Kinder-Wurmfortsatze lassen sich auch am lebenswarm fixierten Säuglingswurmfortsatze dieselben durch Oxyuren verursachten Epitheldefekte nachweisen, und an ihnen sehen wir die ersten entzündlichen Veränderungen, wie wir sie als die häufigsten Anfangsstadien der phlegmonösen Appendicitis kennengelernt haben. An diesem Säuglingswurmfortsatze fehlen jegliche Anzeichen von Stenosen, Verwachsungen oder Kotretentionen. Das Lumen ist, wie die Abbildungen zeigen, weit; der lymphatische Apparat ist noch sehr gering entwickelt und infolgedessen kommen wir zu 
dem zwingenden Schlusse: Das einzig Ausschlaggebende in diesen Wurmfortsätzen für die Entstehung der Appendicitis sind die durch Oxyuren hervorgerufenen Epitheldefekte, und damit ist die Beweiskette der von mir vertretenen Ansicht über die Genese der Appendicitis geschlossen, da ich den in meiner Monographie für den Säuglingswurmfortsatz noch fehlenden Beweis an der Hand des mitgeteilten Falles erbracht habe.

Im Hinblick auf mehrfache Angaben in Referaten über meine Auffassung der Appendicitisgenese, in denen die Behauptung aufgestellt wird, ich sähe in der Oxyuris die alleinige Ursache für die Appendicitis, möchte ich doch darauf hinweisen, daß diese Angaben den in meiner Monographie geäußerten nicht entsprechen. Abgesehen davon, daß die Oxyuris mit der eiterigen Appendicitis direkt überhaupt nichts zu tun hat, berücksichtigen die Referenten nicht, daß ich als vorbereitende Gelegenheitsursache der appendicitischen Eiterung jegliche Noxe in Betracht gezogen habe, die das Epithel des Wurmfortsatzes zerstört, und daß die Genese der Eiterung von Fall zu Fall durch histologische Analyse festzustellen ist, für die auch Stenosen, Kotsteine, Verwachsungen und Abknickungen in Frage kommen können. Daß diese in gewisser Weise von einer auf dem Boden einer früheren Oxyureninfektion entstandenen Eiterung mit ihren Folgezuständen abhängig sein können, ist möglich, aber die oben angeführte Wiedergabe meiner angeblichen Ansichten ist in ihrer Verallgemeinerung irrefübrend. Es wäre doch wohl angebracht, da $B$ man meiner individualisierenden, auf Grund umfangreicher mikroskopischer Untersuchung zustande gekommenen Analyse des appendicitischen Anfalles etwas mehr Rechnung trüge. Dieses vermisse ich auch in einem jüngst erschienenen Aufsatze von Marsch ${ }^{7}$ ). Dieser Autor erwähnt in einer Fußnote zwei akute Appendicitiden mit reichlichen Oxyuren und einem Kotstein im Processus, und ohne mikroskopische Analyse erklärt er einfach den Kotstein für die zur Eiterung führende Ursache. Selbstverständlich ist dies möglich, aber ohne genaue mikroskopische Analyse ist es unmöglich, etwas Sicheres über die Genese der Eiterung auszusagen. Abgesehen davon sind die Möglichkeiten, auf welchem Wege der Kotstein zur Appendicitis führt, ja auch noch verschiedene. Dieses habe ich alles ausführlich in meiner Monographie auseinandergesetzt, durch die Abb. 43, 44, 45, $46,47,48$ und 49 erläutert und betont, da $\beta$ den verschiedenen Möglichkeiten der Pathogenese der Appendicitis früher nicht genügend Berücksichtigung geschenkt wurde. Ganz besonders habe ich dabei eine dem Kotsteine zugeschriebene, die Schleimhaut vor der Entzündung schützende Wirkungsweise an der Hand von Abbildungen widerlegt. Wenn Marsch ohne mikroskopische Untersuchung oder Belege diese Frage jetzt kurz entscheidet, so räumt er der von Fall zu Fall verschiedenen Pathogenese der Eiterung zu geringe Bedeutung ein. 
Der mikroskopische Befund des in Abb. 1 und 2 wiedergegebenen Falles ist mit kurzen Worten folgender: Die Entzündungserscheinungen sind imo wesentlichen noch auf die obersten Abschnitte der Schleimhaut beschränkt. Ich gebe in Abb. 4 die defekte Bucht der Abb. 1 bei starker Vergrößerung wieder, die in Gestalt eines Fibrinnetzwerkes mit leukocytären Einlagerungen die eben beginnende entzündliche Reaktion zeigt. Der in Abb. 2 wiedergegebene Defekt auf der Faltenhöhe

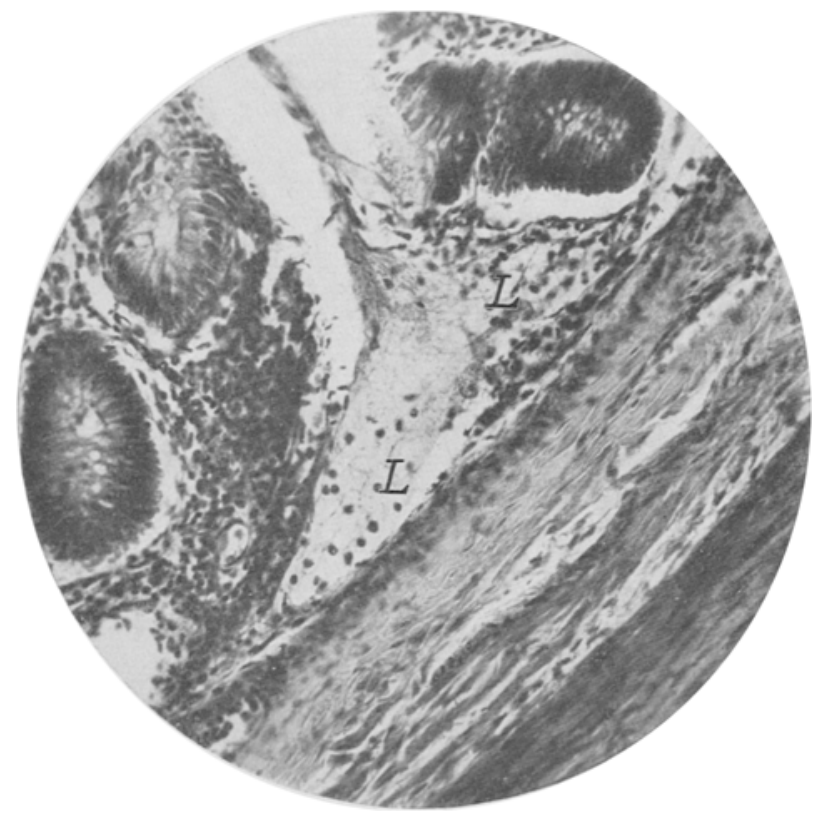

Abb. 4. Processus-Querschnitt. (200 fache Vergrößerung. 3 Monate alter Säugling.) Sekundär infizierter Oxyurendefekt in der Tiefe einer Bucht. Dasselbe Präparat wie Abb. 1. (Die Stelle $\times$ der Abb. 1 bei starker Vergrößerung.) $L=$ Leukocyten, Lymphocyten und Fibrin.

zeigt an der Oberfläche eine mäßige leukocytäre Auflagerung, die sich als Infiltration etwas tiefer in die Schleimhaut erstreckt und hier in ein Lymphknötchen reicht. In diesem findet sich auch etwas Nekrose, anscheinend in gewucherten Reticulum-Endothelien. $\mathrm{Ob}$ der in dem Lymphknötchen sichtbare Spalt ein bei der Entzündung aufgetretener Gewebsspalt oder ein Oxyurendefekt ist, lasse ich offen. Jedenfalls ist die Spaltbildung auf diese Stelle beschränkt. In der Muskulatur und subserös sind die Entzündungserscheinungen nur andeutungsweise vorhanden, insofern hier und da ganz vereinzelte extravasculäre Leukocyten anzutreffen sind. Allerdings finden sich mehrere stärkere lymphocytäre Zellanhäufungen hauptsächlich subserös, was mir im Sinne der in meiner Monographie geäußerten Auffassung spricht, daß hier schon 
vor dem akuten Anfalle, von den Oxyurendefekten aus, Entzündungs. reize die Wurmfortsatzwand getroffen haben können.

Mit kurzen Worten muß ich noch darauf eingehen, daß der Wurmfortsatz ja gelegentlich einer Bruchoperation gewonnen wurde, um dem Einwande zu begegnen, daß wir es hier mit einer eventuellen Einklemmungsfolge zu tun haben. Dagegen spricht erstens mal, daß bei der Operation überraschenderweise jegliche Einklemmungserscheinungen vermißt wurden und hierfür auch im mikroskopischen Präparate jeglicher Anhaltspunkt fehlt. Blutungen fehlen völlig. Es liegt dieser Fall also ganz ähnlich, wie der von Bamberg l. c. mitgeteilte, bei dem sich ebenfalls bei einer zuerst als eingeklemmt angenommenen Hernie bei der Operation eine allerdings schon weiter vorgeschrittene Appendicitis fand.

Die Diagnose der Appendicitis im Säuglingsalter ist, wie ja schon Bamberg ausführte, nicht so einfach, und unter den 13 von Bamberg zusammengestellten Fällen war die Diagnose nur einmal gestellt worden. Es entzieht sich vorläufig unserer Kenntnis, wie oft sich im Säuglingsalter hinter einer Hernie oder noch häufiger, hinter einem Darmkatarrh, eine Appendicitis verbirgt.

Daßs die Bedingungen für einẹn Appendicitisanfall auch im Säuglingsalter gar nicht so selten gegeben sind, möchte ich daraus schließen, daß ich bei einer Anzahl von fünf lebenswarm fixierten Säuglingswurmfortsätzen, von denen drei oxyurenhaltig, waren, in einem der letzteren Epitheldefekte fand, und zwar gerade an der Stelle, wo eine Oxyuris auf Längsschnitten untersucht, dem Epithel anlag. Über die Häufigkeit eines derartigen Befundes gestatten natürlich diese fünf von mir ausgeführten Untersuchungen kein definitives Urteil, da es sich ja um einen Zufallsbefund handeln kann. Immerhin sollte er doch die Veranlassung geben, dieser Frage eine größere Aufmerksamkeit zuzuwenden. Es handelt sich also hier um die in meiner Monographie besprochene Frage der natürlichen Immunität gegenüber Infekten an Epitheldefekten der Wurmfortsatzsehleimhaut.

Interessant ist in dieser Beziehung ein weiterer von mir erhobener Befund am Wurmafortsatze eines $13 / 4$ Jahre alten Kindes, das an epidemischer Genickstarre gestorben war und dessen Wurmfortsatz durch eine gleich nach dem Tode vorgenommene intrabbdominelle Formalininjektion fixiert wurde. Hier fand sich mikroskopisch eine eben beginnende eiterige Appendicitis, hauptsächlich in der Tiefe der Buchten, und zwar an Epitheldefekten, wie wir sie als durch Oxyuren entstanden ansehen müssen. Die Epithelregenerationen waren in diesem Falle nur spärlich, aber in deutlicher Weise vorhanden, und da auch hier im Lumen des Processus Oxyuren vorhanden waren, fügt sich dieser Fall ganz in den Rahmen der in meiner Monographie niedergelegten 
Beobachtungen ein. Klinische Erscheinungen sind in diesem Falle nicht in die Erscheinung getreten, aber das will hier nichts besagen in Anbetracht des zarten Alters des Knaben und der im Vordergrunde stehenden zum Tode führenden Genickstarre.

Ich gebe in Abb. 5 eine Abbilaung der leukocytären Pfropfbildung dieses Falles wieder an einem Epitheldefekt, der weit größer ist als die Ausdehnung der leukocytären Auflagerung. An den verschiedensten Stellen finden sich noch ähnliche derartige Epitheldefekte, die teils

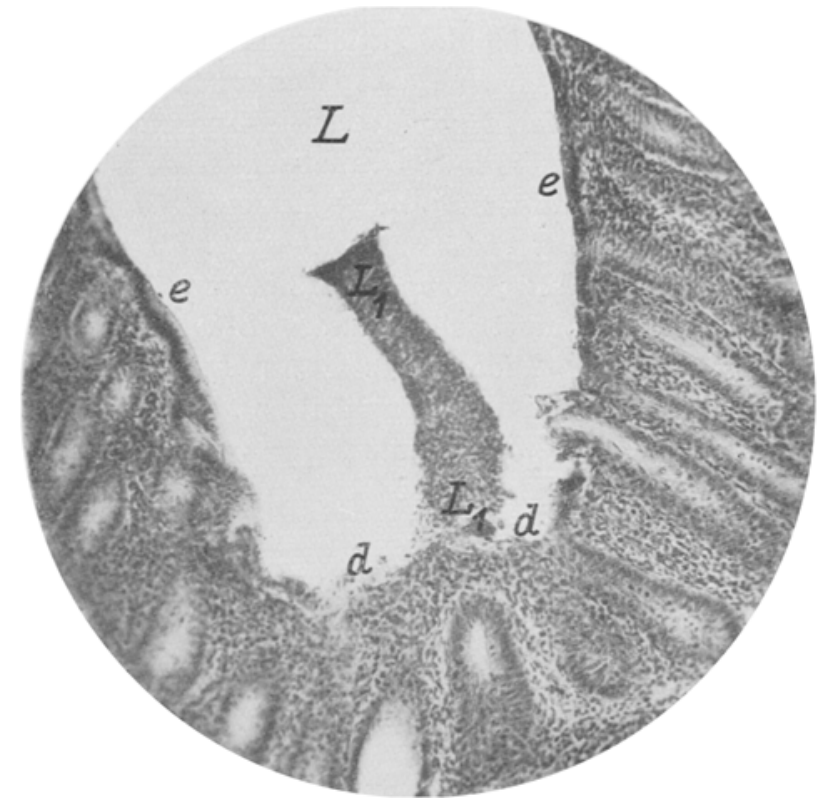

Abb. 5. Processus-Querschnitt. (140 fache Vergrößerung. $1 \frac{3}{4}$ Jahre altes Kind.) $L=$ Iumen des Processue. Bei $e$ erhaltenes Epithel einer Bucht. Am Ende der Bucht bei $d=$ Fpitheldefekt; hier bei $L_{1}=$ Leukocytenpfropf. (Leichenpräparat; lebenswarm fixiert.)

ganz reaktionslos durch alle Übergangsstadien mit den Herden der ausgesprochenen leukocytären Pfropfbildung vesbunden sind. Es ist also hier an den durch Oxyuren verursachten Epitheldefekten stellenweise zu einer sekundären mykotischen Infektion gekommen, und da muß ich mit einigen Worten auf die in meiner Monographie gemachten Ausführungen zurückkommen. Ich erklärte dort auf S. 48, daß es notwendig wäre, darauf zu achten, ob auch schon bei jüngeren Kindern derartige durch Oxyuren verursachte Epitheldefekte vorkämen und ob sie hier seltener zur sekundären Infektion Veranlassung gäben als bei älteren Kindern. Nun hat sich also bei den vorliegenden Untersuchungen die merkwürdige Tatsache ergeben, daß bei 6 wahllos untersuch ten Fällen der beiden ersten Lebensjahre sich in den oxyurenhaltigen 
Wurmfortsätzen eines 6 monatigen Säuglings und eines $13 / 4$ jährigen Knaben Epitheldefekte fanden, und daß letzterer eine sekundäre mykotische Infektion aufwies. Das ist möglicherweise in dem Sinne zu deuten, daß entweder derartige sekundäre Infektionen im frühesten Kindesalter mehr die Neigung zur lokalen Beschränkung zeigen oder aber, daß, wenn sie weiter gehen, diese Infektionen unerkannt zum Tode führen, da ja die Appendicitis vor dem 2. Lebensjahre im allgemeinen als eine relativ seltene Erkrankung angesehen wird. In diesem Zusammenhange ist es vielleicht von Interesse zu erfahren, daß ich unter einer relativ kleinen Anzahl von 20 Kindersektionen im Alter von 2-3 Jahren makroskopisch einen weiteren Fall von eiteriger Appendicitis als zufälligen Nebenbefund feststellen konnte. Die Todeskrankheit war in diesem Falle eine Drüsentuberkulose mit allgemeiner Dissemination und ausgedehnter geschwüriger Darmtuberkulose. Hier sei nur kurz der anatomische Befund am Processus mitgeteilt: $3^{1 / 2}$ jähhriger Knabe. Proc. retrocöcal, ziemlich lang; die untere Hälfte auf der Beckenschaufel verwachsen, distal kolbig aufgetrieben, letztere Stelle weit über bohnengroß. Der aufgeschnittene Processus zeigt im Lumen etwas Schleim und breiig-flüssigen Kot; auf der Schleimhaut zwei eierhaltige Oxyuren. Vor der bohnengroßen Erweiterung unten ist das Lumen stark verengt, die Schleimhaut verdickt. Hinter der Verengerung befindet sich reichlich Eiter im Lumen; die Schleimhaut ist hier größtenteils ulceriert, zum Teil bis auf die Muscularis. Hier steckt noch in der Wand eine dritte weibliche Oxyuris, die mit dem Hinterende ins Lumen hineinragt.

Mikroskopisch zeigte die Wand des Processus im Bereiche des Empyems eine phlegmonöse Appendicitis, die bereits das Bauchfell ergriffen hatte, aber infolge der eingangs erwähnten Verwachsungen war es nicht zu einer allgemeinen Infektion des Bauchfells gekommen. Auf welchem Wege hier die eiterige Appendicitis entstanden war, kann ich bei dem vorgeschrittenen Zustande der Entzündung am Leichenpräparatè nicht angeben, zumal die histologische Analyse durch die kadaverösen Veränderungen erschwert war. Im wesentlichen sind für die Histogenese der Appendicitis in diesem Falle drei Möglichkeiten zu erwägen. Erstens kann es sich um eine Stagnationsappendicitis hinter der oben erwähnten Stenose handeln. Zweitens kann die im Bereiche der Eiterung bestehende Ulceration mit Granulationen tuberkulöser Natur gewesen sein, da sonst im Typhlon und Tleum eine ausgedehnte geschwïrige Tuberkulose bestand. Mikroskopisch fand sich nichts für Tuberkulose Beweisendes, aber das spricht ja nicht unbedingt gegen Tuberkulose, da man öfters neben histologisch kenntlichen tuberkulösen Geschwüren auch solche ohne sichere histologische Zeichen einer tuberkulösen Natur findet, die sich aber öfters durch den Nachweis von Tuberkelbacillen als sicher tuberkulös erweisen. Hier ließen sich 
Beitrag zur Appendicitisfrage beim Säugling und im frühen Kindesalter. 213

Tuberkelbacillen nicht nachweisen. Drittens ist es möglich, daß die hier in der Wand des Processus gefundene Oxyuris bzw. die durch sie hervorgerufene Defektbildung zu einer sekundären Infektion und hierdurch zu der bei der Sektion gefundenen Appendicitis Veranlassung gegeben hat. Wenn ich also die Histogenese dieser Appendicitis offenlasse, so will es mix wichtig erscheinen, daß ich unter einem relativ kleinen Materiale von Appendices, die dem Säuglingsaiter und dem frühen Kindesalter angehörten, und von denen nur ein geringer Bruchteil genau mikroskopisch untersucht wurde, 3 Fälle fand, die alle durch Oxyuren kompliziert waren, in denen sich einmal die beginnende eiterige Appendicitis fand. Im zweiten Falle fand sich eine phlegmonös-ulceröse Appendicitis mit Beteiligung des Bauchfells, und im dritten Falle fanden sich Epitheldefekte bei einem Säugling ohne nennenswerte entzündliche Erscheinungen. In dem Falle, bei dem schon das Bauchfell in Mitleidenschaft gezogen war, ist die Appendicitis ebenfalls ohne klinische Erscheinungen verlaufen, wie ich aus der mir liebenswürdigerweise zur Verfügung gestellten Krankengeschichte ersehe. Aber hieraus wird man keine Schlußfolgerungen ziehen können, da evtl. Symptome durch schwerere, bei der allgemeinen zum Tode führenden tuberkulösen Infektion überlagert gewesen sein können.

Auf die Frage, ob vielleicht akute Infektionskrankheiten oder auch solche chronischer Natur einen Einfluß darauf haben, ob die Oxyuren an der Schleimhaut des Wurmfortsatzes Epithelschädigungen verursachen, möchte ich vorläufig noch nicht eingehen. Ebenso möchte ich die Frage noch nicht beantworten, ob im frühesten Kindesalter die durch Oxyuren hervorgerufenen Epitheldefekte der Schleimhaut seltener sind als im späteren Kindesalter, und zwar hauptsächlich bei solchen Kindern, die akute Entzündungen oder chronische Beschwerden, sog. "Wurmschmerzen", haben, da mein Untersuchungsmaterial vorläufig zur Entscheidung noch nicht ausreicht. Ebenso muß ich die in meiner Monographie aufgeworfene Frage noch unbeantwortet lassen, ob sich vielleicht auf dem Wege der histologischen Analyse ein Unterschied ergibt bei den Wurmfortsätzen, die bei bestehenden Epitheldefekten keine Neigung zur sekundären mykotischen Infektion haben im Gegensatz zu solchen, die bei bestehenden Defekten eine solche Neigung erkennen lassen und ob hierbei vielleicht immunisatorische Eigenschaften von Blutzellen, so z. B. der Eosinophilen, eine Rolle spielen, die ja von Buchner als Alexocyten bezeichnet wurden. Ich werde über diese Untersuchungen berichten, wenn mein Material die gewünschte Reichhaltigkeit aufweisen wird, wofür ja nur lebenswarm fixierte Wurmfortsätze des Leichenmateriales in Betracht kommen. Aber vielleicht tragen diese Ausführungen dazu bei, andere Untersucher auf diese Verhältnisse hinzuweisen und zur Mitarbeit anzuregen. 
Zum Schluß möchte ich noch die Lücke ausfüllen, die sich in meiner Monographie in bezug auf Angaben über die Häufigkeit des Vorkommens von Helminthen im Säuglings- und Kindesalter fand, da mir damals hierzu die nötige Unterlage fehlte. Natürlich beziehen sich diese Angaben jetzt nur auf Groß-Berliner Verhältnisse.

Ich fand bei 154 Kindersektionen bis zu 14 Jahren 62 mal Oxyuren, 2 mal Trichocephalen und nur 1 mal Ascaris, d. h. also:

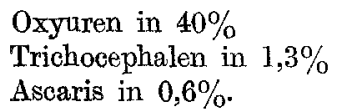

Trichocephalen und Ascaris fanden sich nur nach dem 2. Lebensjahre. Die Oxyuren fanden sich im 1. Lebensjahre in $20 \%$ der Fälle, aber mit dem Unterschiede, daß sie sich im 1. Halbjahre (34. Fälle) in $8,8 \%$ und im 2. Halbjahre (32 Fälle) in 34\% fanden.

Im 2. Lebensjahre fanden sich Oxyuren in $41 \%$; zwischen 2 und 6 Jahren in $59 \%$ und zwischen 6 und 14 Jahren in $74 \%$. Wir sehen also bereits nach der 1. Hälfte des 1. Lebensjahres ein rasches Ansteigen.

Diese Zahlen erwecken den Eindruck, als ob das ganz erheblich höhere Werte sind als die von mir ${ }^{1}$ ) aus dem Jahre 1909 mitgeteilten, wo ich im 1. Lebensjahre $5 \%$, im 2. 10\%, zwischen 2 und 6 Jahren $40 \%$ und zwischen 6 und 14 Jahren $45 \%$ angegeben hatte. Aber dieser Unterschied ist nur ein scheinbarer; denn meine damaligen Angaben bezogen sich nur auf den makroskopischen Befund bei der Sektion, und dieser betrug damals $21,7 \%$ für alle Fälle, und jetzt, wenigstens zuerst bei einer der damaligen analogen Anzahl, 20,9\%. Die jetzigen Zahlen beziehen sich auf makroskopische und mikroskopische Untersuchungen, da ich jeden Processus-Inhalt genau mikroskopisch untersuchte. Daraus schließe ich, daß gegen das Jahr 1909 jetzt keine nennenswerte Vermehrung der Oxyuren in Berlin beim Kinde stattgefunden hat. Der äußerst geringe Prozentsatz der Ascaris erklärt sich dadurch, daß die überwiegende Mehrzahl meiner Fälle den beiden ersten Lebensjahren angehört. Auf das Alter von 2-14 Jahren berechnet, ergibt sich knapp 2\%. Das sind also für Trichocephalen und Ascariden ganz analoge Verhältnisse wie im Jahre 1909, da ich bei einer 3 mal geringeren Anzahl damals nur 1 mal Trichocephalen und keinmal Ascaris fand.

Wenn ich mich in meiner Monographie dahin ausgesprochen habe, daß infolge des Krieges beim Erwachsenen eine Vermehrung der Oxyuren eingetreten ist, so bin ich in dieser Auffassung schwankend geworden. Gewiß ist es möglich, daß eine mäßige Zunahme stattgefunden hat, aber sehr bedeutend kann dieselbe nicht sein. Denn auch jetzt noch ist an der Leiche beim Erwachsenen der Oxyurennachweis häufiger nur bei speziell darauf gerichteter Aufmerksamkeit und sehr oft nur nach mikroskopischer Untersuchung möglich, wie ich ${ }^{8}$ ) erst kürzlich aus- 
führte. In diesem Sinne würden auch meine Befunde aus dem Jahre 1913 sprechen, bei denen ich ja schon bei nur makroskopischer Betrachtung des exstirpierten Erwachsenen-Wurmfortsatzes in $32 \%$ Oxyuren nachweisen konnte gegen $50-60 \%$ nach dem Kriege, wo bei letzteren Untersuchungen eine mikroskopische Kontrolle den Prozentsatz stark ansteigen ließ. Man achtet jetzt eben mehr auf diese Parasiten und deshalb findet man sie häufiger, zumal das Auge jetzt etwas geschulter für diese Spczialfrage geworden ist. Das geht auch aus der Mitteilung Staemmlers (Zentralbl. f. allg. Pathol. 31, H. 15) hervor, der jetzt in Schnitten von im Jahre 1904/05 exstirpierten meist entzündeten Wurmfortsätzen in $28 \%$ Oxyuren nachwies, wobei noch in Betracht zu ziehen ist, daB sein Material meist in aufgeschnittenem Zustande zu seiner Beobachtung kam, wo also sicher schon ein erheblicher Teil der Oxyuren verlorengegangen war.

Nun ist es aber ganz interessant, an der Hand der von mir neuerdings untersuchten Sektionsfälle in bezug auf die klinische Nachweismöglichkeit der Oxyuren Betrachtungen anzustellen. Es ist das ein Punkt, auf den ich leider immer wieder von neuem zurückkommen muß, da er in den klinischen Arbeiten nicht genügend berücksichtigt wird. Ich fand jetzt beim Kinde nach mikroskopischer Untersuchung eines jeden Processus-Inhaltes in Verbindung mit genauer makroskopischer Betrachtung des Darminhaltes folgendes: 11 mal fanden sich 1, 13 mal 2,

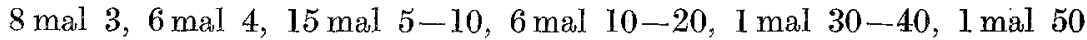
und 1 mal 85 Oxyuren, d. h. also in über der Hälfte der Fälle betrug die Zahl der gefundenen Oxyuren 3 und darunter. Da sich hierunter relativ häufig nur mikroskopisch ein einzelnes Männchen oder nicht eierhaltige weibliche Exemplare fanden, so ist praktisch die Möglichkeit des klinischen Nachweises äuBerst gering oder überhaupt nicht vorhanden. Es ist deshalb auch nicht richtig, wenn $v$. Gottberg $\left.{ }^{9}\right)$ schreibt, daß einzelne Kinder, die Oxyuren beherbergen, bei der Untersuchung als Nicht-Oxyurenträger bezeichnet werden. Es geschieht dies ungefähr bei der Hälfte der Fälle. Wenn die Gesamtzahl der Oxyurenträger nach Prozenten bei Gottberg (32\%) und bei mir (40\%) nicht so sehr differiert, so liegt das wohl daran, daß bei mir das Säuglingsalter allein mit 65, und das 1. Lebensjahr mit 38 Fällen vertreten ist, d. h. zusammen mit über $2 / 3$ der Fälle. Bei Gottberg hat es sich offenbar um ältere Kinder gehandelt; das geht nämlich aus Gottbergs Angabe hervor, daß die Kinder nach evtl. Schmerzen ausgefragt wurden, was bei kleinen Kindern unmöglich ist. Dieses, wie ich meine, scheinbare Freisein von Würmern ist nämlich deshalb von Wichtigkeit, weil ja die Beziehungen unerklärlicher Bauchschmerzen zu Würmern noch keineswegs genau geklärt sind. Unter unerklärlichen Bauchschmerzen verstehe ich solche, bei denen organische Veränderungen oder der Genuß unbekömmlicher 
Lebensmittel, so auch von unreifem Obst oder zu reichlicher Obstgenuß evtl. in Verbindung mit Wassergenuß, keine Rolle spielt. So konnte ich bei Durchsicht der Krankengeschichten der von mir obduzierten Kinder, bei denen organische Veränderungen nicht in Betracht kamen, mehrere Fälle feststellen, die in der Anamnese über Bauchschmerzen oder sog. Nabelkoliken geklagt hatten, und die schon deswegen früher in anderen Krankenanstalten behandelt und als nervös usw. bezeichnet worden waren.

Dafür, daß derartige Bauchschmerzen mit Würmern zusammenhängen können, sprach sich kürzlich auch Finkelstein ${ }^{14}$ ) aus. Dieser Autor sah diese Schmerzen nach antihelminthischen Kuren verschwinden. Allerdings sah Finkelstein einen Unterschied bei den ambulatoriseh behandelten Patienten gegenüber denen aus der Privatpraxis. Ich fand früher in gleicher Weise bei operierten Kindern der III. als auch der II. und I. Klasse, die anamnestisch spontan derartige Bauchschmerzen angaben, Oxyuren in der exstirpierten Appendix. Die Wirkung der in diesen Fällen gelegentlich schon früher verordneten Wurmmittel war von wechselndem Erfolge oder auch Mißerfolge mit längerem oder kürzerem schmerzfreiem Intervalle gewesen. Abgesehen von der Tatsache, daß die Wurmmittel meist die Würmer nicht definitiv vertreiben, sondern nur schädigen, spielt auch der Umstand eine Rolle, daß z. B. Zitwersamen öfters gar kein Santonin enthält, wovon ich mich durch eigene Untersuchungen überzeugt habe. Anderseits liegt auch die Möglichkeit vor, daß es individuell je nach der Füllung des Darmes verschieden wirken kann. Aber es liegt mir ferne leugnen zu wollen, daß bei diesen Schmerzen ohne anatomische Unterlagen gelegentlich neben individueller Überempfindlichkeit gegen gewisse Nahrungsmittel evtl. auch noch Störungen der inneren Sekretion, z. B. der Nebennieren, mit einer möglichen Einwirkung auf das sympathische System möglicherweise eine Rolle spielen können. In meinen Fällen war es nur so auffallend, wie häufig bei den durch Exstirpation des Wurmfortsatzes festgestellten Oxyurenträgern das fragliche Krankheitsbild spontan angegeben wurde. Hier war auch öfters vorher vergeblich nach Oxyuren gesucht worden, und die Kinder galten als Nicht-Oxyurenträger.

Schließlich spielt bei dem wechselnden zeitlichen Auftreten dieser Bauchschmerzen auch der Unterschied der ambulatorisch behandelten Patienten gegenüber denen der Privatpraxis eine Rolle. Denn erstere beobachtet man meist nicht über eine längere Zeit, und man bekommt über evtl: Rezidive wohl längst nicht immer so genaue Angaben wie bei solchen Patienten der Privatpraxis. Auch wird oft den Beschwerden bei Patienten der letzteren Kategorie von seiten der Eltern größere Aufmerksamkeit geschenkt, denn die „Blinddarmfurcht" ist in diesen Kreisen meist größer, und es werden auch geringere Beschwerden mehr beachtet. 
Was nun die Äußerungen Gottbergs ${ }^{9}$ ) anbetrifft, der die Angaben der Eltern dieser Kinder, welche diese Bauchschmerzen mit den Oxyuren in Zusammenhang brachten, ablehnt, so vermisse ich bei Gottberg ein Eingehen auf die in meiner Monographie gemachten Ausführungen, wo diese Fragen an der Hand mitgeteilter Krankengeschichten etwas ausführlicher erörtert wurden. Auch schon in meinem von Gottberg zitierten Aufsatze aus dem Jahre 1914 (Berl. klin. Wochenschr. 26, 27) besprach ich dieses Thema, ohne daß $v$. Gotlberg hierauf eingeht.

Ebenso habe ich in anderen Aufsätzen die Beobachtung machen können, daß meinen Ausführungen in ihrem wesentlichsten Punkte noch keine Aufmerksamkeit gewidmet wird. Das gilt auch z. B. für eine kurze Mitteilung Bönningers ${ }^{10}$ ). Dieser Autor meint, daß es, ebenso wie es eine Immunität gegen den Wanzenbiß gäbe, auch eine solche gegen Oxyuren gäbe. Eine solche Immunität will ich keineswegs bestreiten. Bönninger versucht das an sich selbst durch Erfahrungen, die er im Kriege gemacht hat, zu beweisen, und er führt aus, daß er im Kriege nie vonWanzen gebissen worden sei, obwohl reichlichGelegenheit dazu vorhanden gewesen wäre. Ich halte es hierdurch für bewiesen, daß Bönninger gegen den Wanzenbiß immun ist, nicht aber, daß Bönninger auch gegen. Oxyuren immun ist. Denn. Bönninger hat bei sich im Stuhle gelegentlich vereinzelte Oxyuren beobachtet, und wenn er ohne Behandlung später keine mehr bemerkt hat, so darf Bönninger hieraus nicht schließen, daß er auch gegen Oxyuren immun ist. Denn dadurch, daß Bönninger bei sich Oxyuren im Stuhl bemerkt hat, die sich natürlich in seinem Darme entwickelt haben müssen, beweist Bönninger, da $B$ er nicht gegen Oxyuren immun ist, sondern daß Bönninger höchstwahrscheinlich diejenige geringe Infektion mit Oxyuren aufweist, wie sie in der Mehrzahl der Fälle vorhanden ist, und wie ich sie auf Seite 230 meiner Monographie Schilling gegenüber, als für die Praxis wichtig, besonders betonte.

In einer kurzen Mitteilung, die aber leider gar nicht auf die genaue Histologie der Pathogenese der Appendicitis einging, ist diese Angabe von mir durch Becker ${ }^{11}$ ) jüngst bestätigt worden. Becker schreibt dort Seite 172: „Rheindorfs Behauptung, daß es sich meist um Einzelinfektionen handelt, kann ich durchaus bestätigen."

Wenn also die Autoren bei kïnftigen Veröffentlichungen vielleicht etwas mehr auf die Punkte eingehen würden, die ich in meinen Aufsätzen als wesentlich bewiesen habe, so werden wir eher zu einer Klärung der vorliegenden Probleme kommen. Fs wird das für mich auch das Befreiende haben, daß ich nicht immer wieder vergeblich auf dieselben Punkte zurückkommen muß. Ich würde nicht auf die Bönningersche Behauptung eingegangen sein, wenn nicht in einem kürzlich erschienenen Aufsatze Heubner ${ }^{12}$ ) diese Angaben Bönningers als zu Recht bestehend zitiert hätte, wovon ich eine ungünstige Beeinflussung der dieses Thema 
bearbeitenden Forscher befürehte. Genauer auf diesen Aufsatz Heubners einzugehen, würde mich hier zu weit führen. Nur möchte ich kurz erwähnen, daß, wie ich glaube, Heubner die an und für sich sehr interessanten Beobachtungen an sich selbst etwas zu sehr zu verallgemeinern geneigt ist. In Wirklichkeit liegen für die Praxis die Verhältnisse ganz anders. Denn wie Heubner die Oxyurenfrage bei sich schildert, d. h. die Masseninfektion, liegen die Fälle nur ganz ausnahmsweise; sie verlaufen aber keineswegs, wie ich auf Grund eigener Beobachtungen versichern kann, so wie Heubner es bei sich schildert. Dieser von Heubner angegebene Entwicklungszyklus von 6-7 Wochen Dauer fehlt bei den meisten Fällen völlig, da hier eine nennenswerte Vermehrung überhaupt nicht stattfindet.

Weiter gibt es zum Unterschiede gegen die Heubnerschen Beobachtungen bei den seltenen Masseninfektionen Fälle, die täglich Dutzende and Hunderte von Oxyuren ausscheiden, und weiter solche, bei denen ohne jede Behandlung plötzlich über Monate hinaus keine Würmer mehr nachweisbar sind, die aber'deshalb nicht als oxyurenfrei zu betrachten sind, wie ich mich neulich noch an einem Falle überzeugen konnte, der in seinem exstirpierten Wurmfortsatze einzelne Oxyuren beherbergte. Ws würde mich zu weit führen, hier auf die noch keineswegs geklärte Frage der Oxyurenentwicklung näher einzugehen. In der Beziehung findet sich in der neueren Literatur eine Angabe von Schmidt ${ }^{13}$ ), die von großer Bedeutung ist; dort aber etwas stiefmütterlich behandelt wurde, und welche Bezug hat auf die von mir früher (Frankfurter Zeitschr. f. Pathol. 14, 228) erwähnten Angaben von Vix. Es betrifft das die Weiterentwicklung der Oxyureneier im Darm. Schmidt schreibt dort Seite 39/40 folgendes: „Einmal konnten wir bei dem so aus dem Rectum gewonnenem Schleim, wie auch wiederholt durch das gewöhnliche Analschabsel, teilweise oder ganz entschlüpfte Embryonen in oder neben ihren geplatzten Eihüllen liegend beobachten, ähnlich den Befunden von Vix und Trumpp." Durch derartige Befunde ist natürlich die spontane Fortentwicklung der Oxyureneier im Darme bewiesen, und ich möchte auf die prinzipielle Wichtigkeit derartiger Föllle hier noch besonders hinweisen.

Schlußsätze. 1. An der Hand eines einem 3 Monate alten Säuglinge anläßlich einer Hernienoperation entfernten Wurmfortsatzes, der überraschenderweise mikroskopisch eine frische Appendicitis zeigte, konnte ich nachweisen, daß diese Appendicitis auch von durch Oxyuren verursachten Epitheldefekten ihren Ausgang nahm. Hiermit ist der in meiner Monographie noch fehlende Beweis der Pathogenese der Appendicitis auch für den Säugling erbracht.

2. In fünf lebenswarm fixierten Leichenwurmfortsätzen von Säuglingen fanden sich in einem durch Oxyuren hervorgerufene Epithel- 
Beitrag zur Appendicitisfrage beim Säugling und im frühen Kindesalter. 219

defekte, als Beweis dafür, daß die Bedingungen zum appendicitischen Anfall auch schon beim Säuglinge vorhanden sind. Die Häufigkeit eines derartigen Befundes ist durch weitere Untersuchungen festzustellen.

3. In dem einzigen, gleich nach dem Tode fixierten Processus eines $1^{3} / 4$ Jahre alten, an epidemischer Genickstarre gestorbenen Knaben deckte die mikroskopische Untersuchung als Nebenbefund eine eben beginnende, von Oxyurendefekten ausgehende eiterige Appendicitis auf.

4. Bei einem $3 \frac{1}{2}$ jährigen an Tuberkulose gestorbenen Knaben fand sich in dem oxyurenhaltigen Processus eine phlegmonös-ulceröse, schon auf das Bauchfell übergreifende Appendicitis. Die Feststellung der Pathogenese der Eiterung war hier, da auch Stenosen und evtl. tuberkulöse Geschwüre in Betracht gezogen werden mußten, bei der vorgeschrittenen Eiterung nicht mehr möglich.

5. Diese unter einer relativ kleinen Anzahl von Säuglings- und Kleinkinder-Wurmfortsätzen erhobenen Befunde lassen es wünschenswert erscheinen, den lebenswarm fixierten Leichenwurmfortsätzen von Säuglingen und Kindern in den ersten Lebensjahren eine erhöhtè Aufmerksamkeit zu schenken und sie nach den im vorstehenden Aufsatze auseinandergesetzten Veränderungen zu untersuchen.

\section{Titeraturverzeichnis.}

1) Rheindorf, Beitrag zur Frage der Bedeutung der Oxyuren bei der Wurmfortsatzentzündung der Kinder. Berl. klin. Wochenschr. 1912, Nr. 10 u. 11. 2) Finkelstein, Lehrbuch der Säuglingskrankheiten. Verlag Julius Springer, Berlin 1921. S. 720/21 .- ${ }^{3}$ ) Bamberg, Über Appendicitis bei Säuglingen. Inaug.-Diss. Lejpzig 1905. - 4) Aschoff, Die Wurmfortsatzentzündung. Gustav Fischer, Jena 1908. S. 98. - ${ }^{5}$ ) Rheindorf, Die Wurmfortsatzentzïndung. S. Karger, Berlin 1920. - ${ }^{6}$ ) Suzucki, The Role of Oxyuris vermicularis in the Etiology of App. usw. Surg., Gynecol. a. obstetr. with internat. Abstract. o. Surg. 21, Nr. 6. 1915. - 7) Marsch, Erfahrungen und Resultate an 9000 Appendektomien. Bruns' Beitr. z. klin. Chirurg. 126, H. 1. - $\left.{ }^{8}\right)$ Rheindorf, Mitt. a. d. Grenzgeb. 34. 9) v. Gottberg, Studien über die Zunahme der Häufigkeit von Darmparasiten bei Kindern im Kriege. Arch. f. Kinderheill. 96, H. 3/4. - $\left.{ }^{10}\right)$ Bönninger, Therap. Halbmonatsch. 1921, H. 3. - $\left.{ }^{11}\right)$ Becker, Besteht ein ursächlicher Zusammenhang zw: schen Oxyuren und der akuten Wurmfortsatzen tzündung? Beitr. z. allg. Pathol. u. pathol. Anat. 68. - 12) Heubner, Studien über Oxyuriasis. Jahrb. f. Kinderheilk. 1922. 98. - ${ }^{13}$ ) Schmidt, Welche versehiedenen Methoden zur Diagnostizie rung von Oxyuriasis gibt es usw. Inaug.-Diss. Rostock 1914. _ ${ }^{14}$ ) Finkelstein, Über Nabelkolik der Kinder. Zeitschr. f. ärztl. Frortbild. 1921, Nr. 17. 